\title{
TEMPERATURE DEPENDENCE OF PHOTOFIELD EMISSION FROM TUNGSTEN 〈001〉 DIRECTION
}

\author{
T. RADoŃ AND S. JASKólika \\ Institute of Experimental Physics, University of Wrocław \\ Pl. Maxa Borna 9, 50-204 Wrocław, Poland
}

(Received January 9, 1996)

\begin{abstract}
The existence of a minimum lying by $0.2 \mathrm{eV}$ above the Fermi level, in the band structure of the $\langle 001\rangle$ direction of tungsten, has been confirmed at temperatures of $600 \div 800 \mathrm{~K}$ of the emitter. With increasing temperature a slight rise of the Fermi level in relation to the band structure has been observed.
\end{abstract}

PACS numbers: 79.60.Bm, 79.70.+q, 71.25.Pi

\section{Introduction}

The initiation of the photofield emission method was aimed to investigate the band structure that extends between the vacuum level and the Fermi level [1], which energy range is inaccessible for conventional photoemission. The method was employed to examine the position, in the energy scale, of singular band points such as the extrema lying above and below the Fermi level [2-4]. Recently, the position of the minimum in the energy band lying by approximately $0.2 \mathrm{eV}$ above the Fermi level, which was theoretically predicted for the $\langle 001\rangle$ crystallographic direction of tungsten [5], has been determined [3].

The aim of this brief paper is to demonstrate how temperature considerably affects the filling up of the empty band minimum with electrons and how this is manifested in the photocurrent characteristic. Occasionally a rise of the Fermi level with increasing temperature was noted.

\section{Experimental}

The method of measuring the energy of photofield emission electrons was extensively described in the papers [6,2]. All measurements were carried out using the field emitter tip of tungsten as a sample. The energy of electrons is estimated from the voltage at which a shoulder in the photocurrent characteristics appears. For the voltage the top of the potential barrier, owing to raising the electric field applied, is lowered to approach the energy level of excited electrons, and the excited 
electrons can be emitted over the top of the barrier. The amount of the lowering is to be determined by a separate measurement of the field emission characteristic. In this way the final energy of excited electrons can be determined. It is assumed that the initial energy of an electron is smaller by a value of the quantum energy, $h \nu=1.96 \mathrm{eV}$ of the light applied as it can be done when the quantum energy is small. In view of the fact that the photocurrents are very small, of the order $10^{-13} \div 10^{-11} \mathrm{~A}$, the measurements were carried out in a vacuum whose background pressure was of the order of $10^{-11}$ torr, and the photocurrents were amplified using the phase-sensitive method.

\section{Results and discussion}

It is known that the electron energy levels lying somewhat above the Fermi level are partially occupied by electrons at room temperature. Such electrons were noticed in the photocurrent characteristic curve, which was employed to determine the position of the energy band minimum that lies by approximately $0.2 \mathrm{eV}$ above the Fermi level for the direction $\langle 001\rangle$ of a crystal of tungsten [3]. It appears that the excitation effect of electrons from the minimum has been considerably enhanced upon heating the emitter at a temperature of approximately $700 \mathrm{~K}$.

A characteristic family of photocurrent curves which were taken at different temperatures of the emitter is presented in Fig. 1. For the reciprocal voltage somewhat higher than 18 a shoulder in the photocurrent curves occurs. The greatest shoulder, which is connected with the greatest increase in the photocurrent, is noted in the temperature range $600 \div 800 \mathrm{~K}$, curve 3 and 4 . Such a great increase or hop of the photocurrent is due to the emission of a relatively large number of electrons of similar energy, in this case the electrons which have been excited from the minimum of the energy band. Thus, the raising of the emitter temperature has enabled to obtain a pure experimental effect and to determine the position of the band minimum which has been noticed weakly at room temperature [3]. It should be noted that the photocurrent near the shoulder has increased approximately fourfold with increasing temperature, which is seen from curves 1-3, whereas the general photocurrent increased only about twice. Further rise in the emitter temperature, cf. curve 5 , is followed by the decay of the effect of interest, which is accompanied by the "smoothing" of the curve, probably by more numerous electrons with a higher energy than the energy corresponding to the minimum of the band. The position of the shoulder points coincides in all curves within the small range of reciprocal voltage, the suitable energy range is $0.01 \mathrm{eV}$.

A second set of shoulders, between the reciprocal voltage 15 and 16 (for higher voltages) has been occasionally observed except the shoulders discussed above. These shoulders are associated with the electron emission from the Fermi level and are marked by $E_{\mathrm{F}}$ in Fig. 1. The shoulders shift towards the low voltages (high reciprocal voltages) with increasing temperature, i.e. for curves 1-5. Hence, it is assumed that the Fermi level $E_{\mathrm{F}}$ shifts towards the high energy, or the work function of the $\langle 001\rangle$ plane of tungsten decreases with increasing temperature. The magnitude of the shift, upon heating the emitter from 300 to $1000 \mathrm{~K}$, is estimated to be approximately $0.04 \mathrm{eV}$. This value is in reasonable agreement with the change of work function of tungsten measured by using a different method [7] 


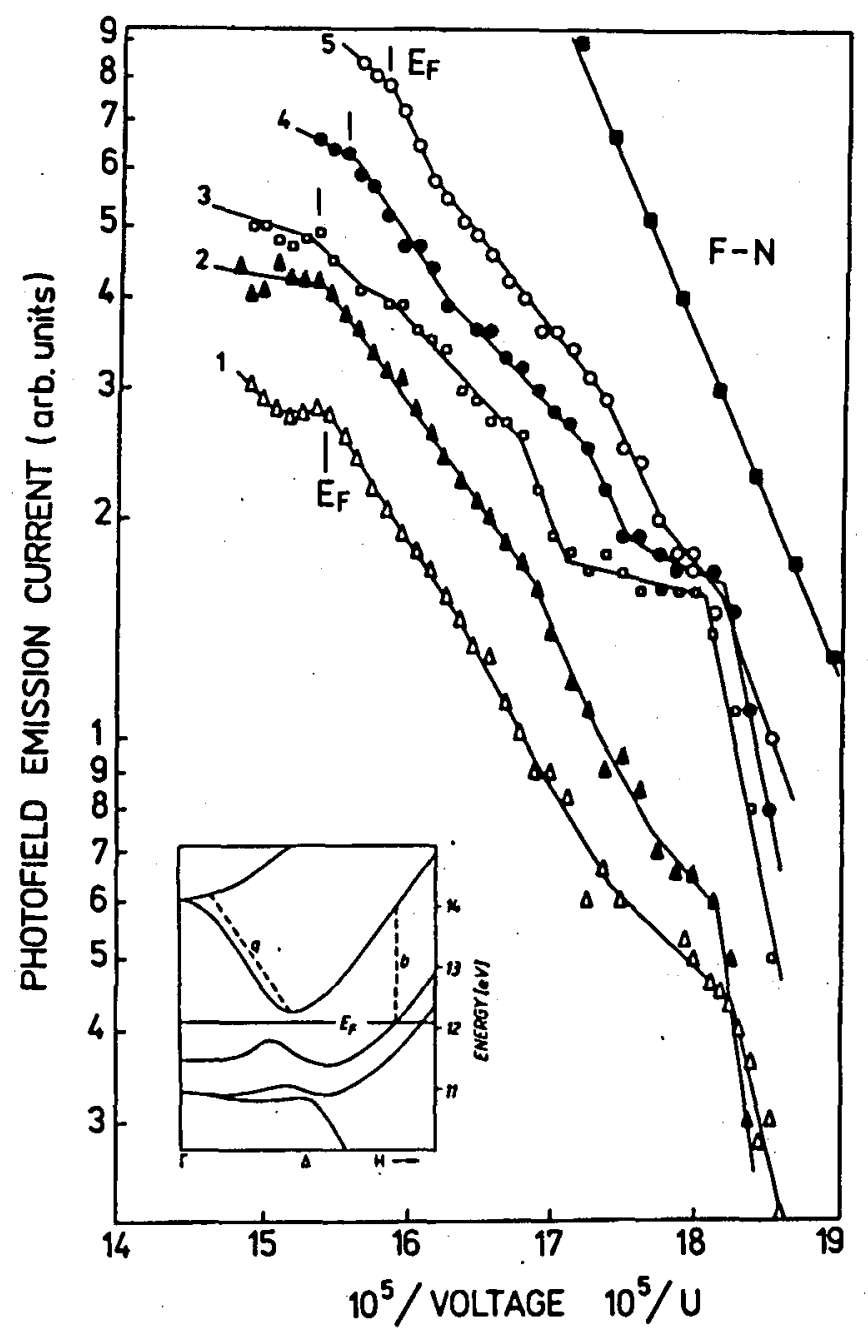

Fig. 1. Dependence of the field emission photocurrent on the reciprocal voltage from the (001) face of a microcrystal of tungsten, as obtained for different temperatures of the field emitter: curve 1 - at $400 \mathrm{~K}, 2$ - at $450 \mathrm{~K}, 3$ - at $600 \mathrm{~K}, 4$ - at $800 \mathrm{~K}$, 5 - at $1050 \mathrm{~K}$. The F-N straight line is a field emission characteristic presented for a qualitative comparison with the photofield characteristics. Part of the band structure of the tungsten $(100)$ direction is included in the figure: a - excitation from the band minimum, $b$ - excitation from the Fermi level.

from which the value of the shift is estimated to be somewhat higher, namely approximately $0.06 \mathrm{eV}$. It should be noted once again that the shoulders in the photocurrent characteristics which were associated with the position of the energy band minimum exhibited no shift in relation to the vacuum level upon changing temperature. 


\section{Acknowledgment}

This work was supported by GBW 94/10/IFD.

\section{References}

[1] H. Neumann, Ch. Kleint, Ann. Phys. (Leipzig) 27, 273 (1971).

[2] T. Radoni, Surf. Sci. 100, 353 (1980).

[3] T. Radoń, S. Jaskółka, Surf. Sci. 231, 160 (1990).

[4] T. Radoń, S. Jaskółka, Surf. Sci. 247, 106 (1991).

[5] N.E. Christensen, B. Feuerbacher, Phys. Rev. B 10, 2349 (1974).

[6] T. Radon', Ch. Kleint, Surf. Sci. 60, 540 (1976).

[7] L.W. Swanson, L.C. Crouser, Phys. Rev. 163, 622 (1967). 